\title{
A QUASI-TRAPEZOID INEQUALITY FOR DOUBLE INTEGRALS
}

\author{
N. S. BARNETT ${ }^{1}$, S. S. DRAGOMIR ${ }^{1}$ and C. E. M. PEARCE ${ }^{2}$
}

(Received 22 January 1999; revised 6 October 2000)

\begin{abstract}
A quasi-trapezoid inequality is derived for double integrals that strengthens considerably existing results. A consonant version of the Grüss inequality is also derived. Applications are made to cubature formulæ and the error variance of a stationary variogram.
\end{abstract}

\section{Introduction}

Although important for applications, numerical integration in two or more dimensions is still a much less developed area than its one-dimensional counterpart, which has been worked on intensively. For some interesting recent commentary, see Sloan [8]. Even the traditional integration of polynomial forms over rectilinear regions translates in higher dimensions to problems with some complications ( $c f$. Rathod and Govinda Rao [6]).

Central to questions of numerical integration in one dimension are Ostrowski's theorem and inequalities of trapezoid type. For a compendious treatment of the latter see Mitrinović et al. [5] and the references therein. Recently new versions of some of the classical tools have been developed for a two-dimensional context.

Suppose $f(\cdot, \cdot)$ is integrable on $[a, b] \times[c, d]$ and for $x \in[a, b]$ and $y \in[c, d]$ set

$$
\begin{aligned}
f^{\dagger}(x, y):= & \int_{a}^{b} \int_{c}^{d} f(s, t) d s d t+(b-a)(d-c) f(x, y) \\
& -(b-a) \int_{c}^{d} f(x, t) d t-(d-c) \int_{a}^{b} f(s, y) d s .
\end{aligned}
$$

\footnotetext{
${ }^{1}$ School of Communications and Informatics, Victoria University of Technology, PO Box 14428, Melbourne City MC, VIC 8001, Australia. E-mail address: \{neil, sever) @matilda.vu.edu.au ${ }^{2}$ Department of Applied Mathematics, The University of Adelaide, Adelaide SA 5005, Australia. E-mail address: cpearce@maths.adelaide.edu.au (c) Australian Mathematical Society 2003, Serial-fee code 1446-1811/03
} 
Barnett and Dragomir [1] have proved the following two-dimensional theorem of Ostrowski type.

THEOREM A. If $f(\cdot, \cdot)$ is continuous on $[a, b] \times[c, d]$ and $f_{x, y}^{\prime \prime}=\partial^{2} f / \partial x \partial y$ exists on $(a, b) \times(c, d)$ and is bounded, that is,

$$
\left\|f_{s, t}^{\prime \prime}\right\|_{\infty}:=\sup _{(x, y) \in(a, b) \times(c, d)}\left|\frac{\partial^{2} f(x, y)}{\partial x \partial y}\right|<\infty,
$$

then for any $x \in[a, b]$ and $y \in[c, d]$

$$
\left|f^{\dagger}(x, y)\right| \leq\left[\frac{(b-a)^{2}}{4}+\left(x-\frac{a+b}{2}\right)^{2}\right]\left[\frac{(d-c)^{2}}{4}+\left(y-\frac{c+d}{2}\right)^{2}\right]\left\|f_{s, t}^{\prime \prime}\right\|_{\infty} \text {. }
$$

Here and subsequently it is implicit that $f_{s, r}^{\prime \prime}$ is integrable on $[a, b] \times[c, d]$.

An interesting particular case, which is in fact the best inequality we can obtain from (1.1), is the 'quasi-midpoint' inequality

$$
\left|f^{\dagger}((a+b) / 2,(c+d) / 2)\right| \leq(1 / 16)(b-a)^{2}(d-c)^{2}\left\|f_{s, t}^{\prime \prime}\right\|_{\infty} .
$$

The first two authors have applied (1.1) to cubature formulæ in [1] and to the analysis of variograms in [2].

Define the functional

$$
\begin{aligned}
f^{*}:= & {\left[f^{\dagger}(a, c)+f^{\dagger}(a, d)+f^{\dagger}(b, c)+f^{\dagger}(b, d)\right] / 4 } \\
= & \int_{a}^{b} \int_{c}^{d} f(s, t) d t d s+\frac{f(a, c)+f(a, d)+f(b, c)+f(b, d)}{4}(b-a)(d-c) \\
& -(d-c) \int_{a}^{b} \frac{f(s, c)+f(s, d)}{2} d s-(b-a) \int_{c}^{d} \frac{f(a, t)+f(b, t)}{2} d t .
\end{aligned}
$$

When Theorem $\mathrm{A}$ applies, we have

$$
\left|f^{\dagger}(a, c)\right| \leq(1 / 4)(b-a)^{2}(d-c)^{2}\left\|f_{s, t}^{\prime \prime}\right\|_{\infty}
$$

and similarly for $f^{\dagger}(a, d), f^{\dagger}(b, c)$ and $f^{\dagger}(b, d)$, so that

$$
\left|f^{*}\right| \leq(1 / 4)(b-a)^{2}(d-c)^{2}\left\|f_{s, t}^{\prime \prime}\right\|_{\infty} .
$$

In this article we show that a much stronger result holds, namely the following.

THEOREM 1. Under the conditions of Theorem A,

$$
\left|f^{*}\right| \leq \frac{1}{16}(b-a)^{2}(d-c)^{2}\left\|f_{s, t}^{\prime \prime}\right\|_{\infty} .
$$

This we establish in Section 2, where it is shown that it follows from an appropriate double-integral identity. In Section 3 we derive a conformable inequality of Grüss type and in Section 4 apply our ideas to cubature formulæ. We conclude in Section 5 with an application to bounds on the error variance of a continuous stream with stationary variogram. 


\section{Integral identities}

First we derive a useful ancillary result.

LEMMA 1. Suppose that $\alpha_{1}<\alpha_{2}$ and $\beta_{1}<\beta_{2}$ and that $\partial^{2} f / \partial s \partial$ t is integrable on $\left[\alpha_{1}, \alpha_{2}\right] \times\left[\beta_{1}, \beta_{2}\right]$. If either $\left(\alpha, \alpha^{\prime}\right)=\left(\alpha_{1}, \alpha_{2}\right)$ or $\left(\alpha^{\prime}, \alpha\right)=\left(\alpha_{1}, \alpha_{2}\right)$ and similarly for $\beta, \beta^{\prime}$, then

$$
\begin{aligned}
\int_{\alpha_{1}}^{\alpha_{2}} \int_{\beta_{1}}^{\beta_{2}}(s-\alpha)(t-\beta) f_{s, t}^{\prime \prime} d t d s & \\
= & \left(\alpha_{2}-\alpha_{1}\right)\left(\beta_{2}-\beta_{1}\right) f\left(\alpha^{\prime}, \beta^{\prime}\right)-\left(\beta_{2}-\beta_{1}\right) \int_{\alpha_{1}}^{\alpha_{2}} f\left(s, \beta^{\prime}\right) d s \\
& \quad-\left(\alpha_{2}-\alpha_{1}\right) \int_{\beta_{1}}^{\beta_{2}} f\left(\alpha^{\prime}, t\right) d t+\int_{\alpha_{1}}^{\alpha_{2}} \int_{\beta_{1}}^{\beta_{2}} f(s, t) d t d s .
\end{aligned}
$$

PROOF. This is immediate from a repeated integration by parts.

We now proceed to our main double-integral identity.

THEOREM 2. Under the assumptions of Theorem A,

$$
f^{*}=\int_{a}^{b} \int_{c}^{d}\left(s-\frac{a+b}{2}\right)\left(t-\frac{c+d}{2}\right) f_{s, t}^{\prime \prime}(s, t) d t d s
$$

PROOF. Take $x \in[a, b], y \in[c, d]$ and apply Lemma 1 with the four choices

$$
\begin{aligned}
& \left(\alpha_{1}, \alpha_{2}, \beta_{1}, \beta_{2}, \alpha, \beta\right) \\
& \quad=(a, x, c, y, a, c),(a, x, y, d, a, d),(x, b, y, d, b, d),(x, b, c, y, b, c) .
\end{aligned}
$$

Addition of the resultant identities yields

$$
\begin{array}{rl}
\int_{a}^{b} \int_{c}^{d} & p(x, s) q(y, t) f_{s, t}^{\prime \prime} d t d s \\
= & (d-c)(b-a) f(x, y)-(d-c) \int_{a}^{b} f(s, y) d s \\
& \quad-(b-a) \int_{c}^{d} f(x, t) d t+\int_{a}^{b} \int_{c}^{d} f(s, t) d t d s,
\end{array}
$$

where $p(x, s)$ is defined as $s-a$ if $s \in[a, x]$ and as $s-b$ if $s \in(x, b]$, whilst $q(y, t)$ is $t-c$ if $t \in[c, y]$ and $t-d$ if $t \in(y, d]$.

We now make the four choices

$$
(x, y)=(a, c),(b, c),(a, d),(b, d)
$$


and add again to derive

$$
\begin{aligned}
\int_{a}^{b} \int_{c}^{d} & {[p(a, s)+p(b, s)][q(c, t)+q(d, t)] f_{s, t}^{\prime \prime}(s, t) d t d s } \\
= & 4 \int_{a}^{b} \int_{c}^{d} f(s, t) d t d s \\
& +[f(a, c)+f(a, d)+f(b, c)+f(b, d)](b-a)(d-c) \\
& -2(d-c) \int_{a}^{b}[f(s, c)+f(s, d)] d s-2(b-a) \int_{c}^{d}[f(a, t)+f(b, t)] d t
\end{aligned}
$$

Since

$$
p(a, s)+p(b, s)=2 s-(a+b), \quad q(c, t)+q(d, t)=2 t-(c+d),
$$

this is equivalent to the desired identity.

Our theorem provides

$$
\left|f^{*}\right| \leq \int_{a}^{b} \int_{c}^{d}\left|s-\frac{a+b}{2}\right|\left|t-\frac{c+d}{2}\right| f_{s, t}^{\prime \prime}(s, t) d t d s
$$

and a simple calculation yields

$$
\int_{\alpha}^{\beta}\left|u-\frac{\alpha+\beta}{2}\right| d u=\frac{(\beta-\alpha)^{2}}{4} .
$$

Theorem 1 follows as an immediate corollary.

\section{An inequality of Grüss type}

The well-known Grüss inequality (see for example Mitrinović et al. [4, p. 296]) states that if $f, g:[a, b] \rightarrow \mathbf{R}$ are integrable on $[a, b]$ and

$$
\varphi \leq f(x) \leq \Phi, \quad \gamma \leq g(x) \leq \Gamma \quad \text { for all } s \in[a, b],
$$

then

where

$$
|I| \leq \frac{1}{4}(b-a)^{2}(\Gamma-\gamma)(\Phi-\varphi),
$$

$$
I:=(b-a) \int_{a}^{b} f(x) g(x) d x-\int_{a}^{b} f(x) d x \int_{a}^{b} g(x) d x .
$$

Moreover, the constant $1 / 4$ is best possible.

We establish a closely related result. 
THEOREM 3. Suppose $f, g:[a, b] \rightarrow \mathbf{R}$ are continuous on $[a, b]$, differentiable on $(a, b)$ and with bounded derivatives. Put

$$
\left\|f^{\prime}\right\|_{\infty}:=\sup _{t \in(a, b)}\left|f^{\prime}(t)\right|<\infty, \quad\left\|g^{\prime}\right\|_{\infty}:=\sup _{t \in(a, b)}\left|g^{\prime}(t)\right|<\infty
$$

Then

$$
\begin{aligned}
\mid I+ & {[f(a)-f(b)][g(a)-g(b)](b-a)^{2} / 4 \mid } \\
\leq & \frac{(b-a)^{2}}{2}\left[\|f-f(a)\|_{\infty}\|g-g(a)\|_{\infty}+\|f-f(b)\|_{\infty}\|g-g(b)\|_{\infty}\right] \\
& +\frac{(b-a)^{4}}{16}\left\|f^{\prime}\right\|_{\infty}\left\|g^{\prime}\right\|_{\infty} .
\end{aligned}
$$

PRoof. Define $h:[a, b]^{2} \rightarrow \mathbf{R}$ by $h(s, t)=[f(s)-f(t)][g(s)-g(t)]$. We have

$$
h(a, a)+h(a, b)+h(b, a)+h(b, b)=2[f(b)-f(a)][g(b)-g(a)]
$$

and

$$
\begin{aligned}
\int_{a}^{b}[ & h(s, a)+h(s, b)] d s \\
\quad= & \int_{a}^{b}[h(a, s)+h(b, s)] d s \\
\quad= & \int_{a}^{b}\{[f(s)-f(a)][g(s)-g(a)]+[f(b)-f(s)][g(b)-g(s)]\} d s .
\end{aligned}
$$

Also

so that

$$
\frac{\partial^{2} h(s, t)}{\partial s \partial t}=-f^{\prime}(s) g^{\prime}(t)-f^{\prime}(t) g^{\prime}(s)
$$

$$
\begin{aligned}
\int_{a}^{b} & \int_{a}^{b}\left(s-\frac{a+b}{2}\right)\left(t-\frac{a+b}{2}\right) \frac{\partial^{2} h(s, t)}{\partial s \partial t} d t d s \\
= & -2 \int_{a}^{b}\left(s-\frac{a+b}{2}\right) f^{\prime}(s) d s \int_{a}^{b}\left(t-\frac{a+b}{2}\right) g^{\prime}(t) d t
\end{aligned}
$$

Hence applying Theorem 2 to $h$ on $[a, b] \times[a, b]$ provides

$$
\begin{aligned}
\int_{a}^{b} \int_{a}^{b}[f(s)-f(t)][g(s)-g(t)] d s d t+\frac{[f(b)-f(a)][g(b)-g(a)]}{2}(b-a)^{2} \\
=(b-a) \int_{a}^{b}\{[f(s)-f(a)][g(s)-g(a)]+[f(b)-f(s)][g(b)-g(s)]\} d s \\
\quad-2 \int_{a}^{b}\left(s-\frac{a+b}{2}\right) f^{\prime}(s) d s \int_{a}^{b}\left(t-\frac{a+b}{2}\right) g^{\prime}(t) d t
\end{aligned}
$$


Since

$$
\frac{1}{2} \int_{a}^{b} \int_{a}^{b}[f(s)-f(t)][g(s)-g(t)] d s d t=I
$$

we deduce that

$$
\begin{aligned}
I+ & \frac{[f(b)-f(a)][g(b)-g(a)]}{4}(b-a)^{2} \\
= & \frac{b-a}{2} \int_{a}^{b}\{[f(s)-f(a)][g(s)-g(a)]+[f(b)-f(s)][g(b)-g(s)]\} d s \\
& -\int_{a}^{b}\left(s-\frac{a+b}{2}\right) f^{\prime}(s) d s \int_{a}^{b}\left(t-\frac{a+b}{2}\right) g^{\prime}(t) d t .
\end{aligned}
$$

Thus the left-hand side of (3.1) is bounded above by

$$
\begin{gathered}
\frac{1}{2}(b-a)^{2}\left[\|f-f(a)\|_{\infty}\|g-g(a)\|_{\infty}+\|f(b)-f\|_{\infty}\|g(b)-g\|_{\infty}\right] \\
+\left\|f^{\prime}\right\|_{\infty}\left\|g^{\prime}\right\|_{\infty}\left[\int_{a}^{b}\left|s-\frac{a+b}{2}\right| d s\right]^{2} .
\end{gathered}
$$

The desired result follows from (2.2).

\section{Application to cubature formulæ}

Take arbitrary divisions $I_{n}: a=x_{0}<x_{1}<\cdots<x_{n-1}<x_{n}=b$ of $[a, b]$ and $J_{m}: c=y_{0}<y_{1}<\cdots<y_{m-1}<y_{m}=d$ of $[c, d]$ and set $h_{i}:=x_{i+1}-x_{i}$ $(i=0, \ldots, n-1)$ and $l_{j}:=y_{j+1}-y_{j}(j=0, \ldots, m-1)$. Define

$$
\begin{aligned}
\eta_{i, j}:= & h_{i} \int_{y_{j}}^{y_{j+1}} f\left(\frac{x_{i}+x_{i+1}}{2}, t\right) d t+l_{j} \int_{x_{i}}^{x_{i+1}} f\left(s, \frac{y_{j}+y_{j+1}}{2}\right) d s \\
& -h_{i} l_{j} f\left(\frac{x_{i}+x_{i+1}}{2}, \frac{y_{j}+y_{j+1}}{2}\right) .
\end{aligned}
$$

Barnett and Dragomir [1] considered a quasi-midpoint rule for double integrals given by

$$
C_{M}\left(f, I_{n}, I_{m}\right):=\sum_{i=0}^{n-1} \sum_{j=0}^{m-1} \eta_{i, j}
$$

and proved that provided the integrals involved exist and $\left\|f_{s, l}^{\prime \prime}\right\|_{\infty}$ is finite, then

$$
\int_{a}^{b} \int_{c}^{d} f(s, t) d s d t=C_{M}\left(f, I_{n}, J_{m}\right)+R_{M}\left(f, I_{n}, J_{m}\right)
$$


where the remainder satisfies

$$
\left|R_{M}\left(f, I_{n}, J_{m}\right)\right| \leq \frac{1}{16}\left\|f_{s, t}^{\prime \prime}\right\|_{\infty} \sum_{i=0}^{n-1} h_{i}^{2} \sum_{j=0}^{m-1} l_{j}^{2} .
$$

We are now able to establish a quasi-trapezoid formula. Set

$$
\begin{aligned}
\xi_{i, j}:= & h_{i} \int_{y_{j}}^{y_{j+1}}\left[\frac{f\left(x_{i}, t\right)+f\left(x_{i+1}, t\right)}{2}\right] d t+l_{j} \int_{x_{j}}^{x_{j+1}}\left[\frac{f\left(s, y_{j}\right)+f\left(s, y_{j+1}\right)}{2}\right] d s \\
& -h_{i} l_{j}\left[\frac{f\left(x_{i}, y_{j}\right)+f\left(x_{i}, y_{j+1}\right)+f\left(x_{i+1}, y_{j}\right)+f\left(x_{i+1}, y_{j+1}\right)}{4}\right]
\end{aligned}
$$

and define

$$
C_{T}\left(f, I_{n}, J_{m}\right):=\sum_{i=0}^{n-1} \sum_{j=0}^{m-1} \xi_{i, j} .
$$

Then we have the following result.

THEOREM 4. Let $f:[a, b] \times[c, d] \rightarrow \mathbf{R}$ satisfy the conditions of Theorem $\mathrm{A}$. Then we have the cubature formula

$$
\int_{a}^{b} \int_{c}^{d} f(s, t) d s d t=C_{T}\left(f, I_{n}, J_{m}\right)+R_{T}\left(f, I_{n}, J_{m}\right),
$$

where the remainder term satisfies

$$
\left|R_{T}\left(f, I_{n}, J_{m}\right)\right| \leq \frac{1}{16}\left\|f_{s, t}^{\prime \prime}\right\|_{\infty} \sum_{i=0}^{n-1} h_{i}^{2} \sum_{j=0}^{m-1} l_{j}^{2} .
$$

PROOF. Applying Theorem 1 to the interval $\left[x_{i}, x_{i+1}\right] \times\left[y_{j}, y_{j+1}\right]$ for $i=0, \ldots$, $n-1$ and $j=0, \ldots, m-1$ gives

$$
\left|\int_{x i}^{x_{i+1}} \int_{y_{j}}^{y_{j+1}} f(s, t) d t d s-\xi_{i, j}\right| \leq \frac{1}{16} h_{i}^{2} l_{j}^{2}\left\|f_{s, t}^{\prime \prime}\right\|_{\infty} .
$$

Summing over $i$ from 0 to $n-1$ and $j$ from 0 to $m-1$ and using the generalized triangle inequality yields the desired inequality (4.1).

REMARK 1. Set

$$
\nu(h):=\max \left\{h_{i}: i=0, \ldots, n-1\right\}, \quad \mu(l):=\max \left\{l_{j}:=j=0, \ldots, m-1\right\} .
$$

Then since

$$
\sum_{i=0}^{n-1} h_{i}^{2} \leq v(h) \sum_{i=0}^{n-1} h_{i}=(b-a) v(h)
$$


and

$$
\sum_{j=0}^{m-1} l_{j}^{2} \leq \mu(l) \sum_{j=0}^{m-1} l_{j}=(d-c) \mu(l),
$$

the right-hand side of (4.1) is bounded above by

$$
\frac{1}{16}\left\|f_{s, 1}^{\prime \prime}\right\|_{\infty}(b-a)(d-c) v(h) \mu(l),
$$

which is of order two precision.

\section{The error variance of a continuous stream with stationary variogram}

Suppose $(X(t))$ is a continuous-time stochastic process, possibly nonstationary. Typically $(X(t))$ represents a continuous-stream industrial process such as is common in many areas of the chemical industry. In [3], the authors considered $X(t)$ as defining the quality of a product at time $t$. The paper was concerned with issues related to sampling the stream with a view to estimating the mean quality $\bar{X}$ characteristic of the flow over the interval $[0, d]$. The sampling location $t$ is said to be optimal if it minimizes the estimation error variance

$$
E\left[(\bar{X}-X(t))^{2}\right], \quad 0<t<d .
$$

In [3] it was shown that for constant stream flows, the optimal sampling point is the midpoint of $[0, d]$ for the situation where the process variogram

$$
\begin{gathered}
V(u)=\frac{1}{2} E\left[(X(t)-X(t+u))^{2}\right], \\
V(0)=0, \quad V(-u)=V(u), \quad u \in[-d, d]
\end{gathered}
$$

is stationary. We remark that variogram stationarity is not equivalent to process stationarity.

In this paper we use Theorem 1 to give an approximation of the estimation error variance $E\left[(\bar{X}-X(t))^{2}\right]$ for $t=d$.

From [3], it can be shown using an identity given in [7] that

$$
\begin{aligned}
E\left[(\bar{X}-X(t))^{2}\right]= & -\frac{1}{d^{2}} \int_{0}^{d} \int_{0}^{d} V(v-u) d u d v \\
& +\frac{2}{d}\left\{\int_{0}^{t} V(u) d u+\int_{0}^{d-t} V(u) d u\right\}
\end{aligned}
$$

Suppose $V$ is continuous on $[-d, d]$, twice differentiable on $(-d, d)$ and has bounded second derivative $V^{\prime \prime}$ bounded on that interval. It is shown in [2] that from 
(1.1) it is possible to get the bound

$$
E\left[(\bar{X}-X(t))^{2}\right] \leq\left[\frac{1}{4}+\frac{(t-d / 2)^{2}}{d^{2}}\right]^{2} d^{2}\left\|V^{\prime \prime}\right\|_{\infty}
$$

for all $t \in[0, d]$.

The best inequality we can get from (5.2) is for $t=t_{0}=d / 2$ when we have the bound

For $t=d$,

$$
E\left[(\bar{X}-X(d / 2))^{2}\right] \leq \frac{d^{2}}{16}\left\|V^{\prime \prime}\right\|_{\infty}
$$

$$
E\left[(\bar{X}-X(d))^{2}\right] \leq \frac{d^{2}}{4}\left\|V^{\prime \prime}\right\|_{\infty}
$$

This can be complemented as follows.

Put $f(s, t)=V(s-t), a=c=0$ and $b=d$ in Theorem 1 to get

$$
\begin{aligned}
& \mid \int_{0}^{d} \int_{0}^{d} V(s-t) d s d t+\frac{V(0)+V(-d)+V(d)+V(0)}{4} d^{2} \\
& \quad-d \int_{0}^{d} \frac{V(-t)+V(d-t)}{2} d t-d \int_{0}^{d} \frac{V(s)+V(s-d)}{2} d s \mid \leq \frac{d^{4}}{16}\left\|V^{\prime \prime}\right\|_{\infty} .
\end{aligned}
$$

Since $V(0)=0$ and $V(-d)=V(d)$, we have

$$
\begin{aligned}
\int_{0}^{d} \frac{V(-t)+V(d-t)}{2} d t & =\int_{0}^{d} \frac{V(s)+V(s-d)}{2} d s \\
& =\int_{0}^{d} \frac{V(t)+V(d-t)}{2} d t=\int_{0}^{d} V(u) d u
\end{aligned}
$$

and by (5.3)

$$
\left|\int_{0}^{d} \int_{0}^{d} V(s-t) d s d t-2 d \int_{0}^{d} V(u) d u+\frac{V(d)}{2} d^{2}\right| \leq \frac{d^{4}}{16}\left\|V^{\prime \prime}\right\|_{\infty} .
$$

But, by the identity (5.1), we deduce that

$$
\int_{0}^{d} \int_{0}^{d} V(s-t) d s d t-2 d \int_{0}^{d} V(u) d u=-d^{2} E\left[(\bar{X}-X(d))^{2}\right] .
$$

Consequently, by (5.4), we get

$$
\left|E\left[(\bar{X}-X(d))^{2}\right]-\frac{V(d)}{2}\right| \leq \frac{d^{2}}{16}\left\|V^{\prime \prime}\right\|_{\infty}
$$

which gives an approximation for $E\left[(\bar{X}-X(d))^{2}\right]$ in terms of $V(d)$. 
Note that for small $d$ the approximation is accurate and is of order two precision.

\section{References}

[1] N. S. Barnett and S. S. Dragomir, "An Ostrowski type inequality for double integrals and applications to cubature formulae", Soochow J. Math., to appear.

[2] N. S. Barnett and S. S. Dragomir, "A note on bounds for the estimation error variance of a continuous stream with stationary variogram", J. KSIAM 2 (1998) 49-56.

[3] N. S. Barnett, I. S. Gomm and L. Armour, "Location of the optimal sampling point for the quality assessment of continuous streams", Austral. J. Stat. 37 (1995) 145-152.

[4] D. S. Mitrinović, J. E. Pečarić and A. M. Fink, Classical and new inequalities in analysis (Kluwer, Dordrecht, 1993).

[5] D. S. Mitrinović, J. E. Pečarić and A. M. Fink, Inequalities for functions and their integrals and derivatives (Kluwer, Dordrecht, 1994).

[6] H. T. Rathod and H. S. Govinda Rao, "Integration of trivariate polynomials over linear polyhedra in Euclidean three-dimensional space”, J. Austral. Math. Soc. Ser. B 39 (1998) 355-385.

[7] I. W. Saunder, G. K. Robinson, T. Lwin and R. J. Holmes, "A simplified variogram method for the estimation error variance in sampling from continuous stream", Internat. J. Mineral Processing 25 (1989) 175-198.

[8] I. H. Sloan, "Multiple integration is intractable but not hopeless", ANZIAM J. 42 (2000) 3-8. 